\title{
Mechanical Properties of Membranes of Poly(L-co-DL-lactic acid) with Poly(caprolactone triol) and Study In Vivo
}

\author{
Marcia Adriana Tomaz Duarte, ${ }^{1,2}$ Adriana Cristina Motta, ${ }^{1}$ \\ and Eliana Aparecida de Rezende Duek ${ }^{1,3,4}$ \\ ${ }^{1}$ Department of Physiological Sciences, Faculty of Medical Sciences, Pontifical Catholic University of São Paulo (PUC-SP), \\ 18030-095 Sorocaba, SP, Brazil \\ ${ }^{2}$ University Center SOCIESC of Santa Catarina (UNISOCIESC), 89206-001 Joinville, SC, Brazil \\ ${ }^{3}$ Department of Materials Engineering, Faculty of Mechanical Engineering, State University of Campinas (UNICAMP), \\ 13083-970 Campinas, SP, Brazil \\ ${ }^{4}$ Departamento de Ciências Fisiológicas, Centro de Ciências Médicas e Biológicas, \\ Pontifícia Universidade Católica de São Paulo (CCMB/PUC-SP), Praça Dr. José Ermírio de Moraes 290, \\ Jardim Vergueiro, CP 1570, 18030-095 Sorocaba, SP, Brazil
}

Correspondence should be addressed to Eliana Aparecida de Rezende Duek; eliduek@fem.unicamp.br

Received 11 September 2014; Revised 19 November 2014; Accepted 21 November 2014; Published 31 December 2014

Academic Editor: Changsheng Zhao

Copyright (C) 2014 Marcia Adriana Tomaz Duarte et al. This is an open access article distributed under the Creative Commons Attribution License, which permits unrestricted use, distribution, and reproduction in any medium, provided the original work is properly cited.

\begin{abstract}
There is increasing interest in aliphatic polyesters from lactones and lactides because of their biodegradability and biocompatibility. Among these compounds, poly(lactide), and poly(glycolide), poly( $\varepsilon$-caprolactone) and their copolymers are especially interesting because of their potential applications as biomedical materials. The aim of this study was to examine the properties of membranes of poly(L-co-D,L lactic acid) (PLDLA) with poly(caprolactone triol) (PCL-T) obtained by solvent evaporation. The blends were characterized by differential scanning calorimetry, dynamic mechanical analysis, Fourier transform infrared spectroscopy, and tensile strength tests. Based on the results of in vitro studies, PLDLA/PCL-T blends of 100/0 and 90/10 were implanted in subcutaneous tissue of Wistar rats for 1, 3, 7, 15, and 60 days to evaluate their biocompatibility. Histological analysis indicated that, although PCL-T-containing membranes caused a more prominent inflammatory reaction in the initial time intervals, by 60 days after implantation, the material was surrounded by dense, organized collagen with almost no inflammatory infiltrate.
\end{abstract}

\section{Introduction}

The biomaterials are meant to be implanted in the human body as constituents of devices that are designed to perform certain biological functions by substituting or repairing different tissues such as bone, cartilage or ligaments, and tendons and even by guiding bone repair when necessary. For this reason, polymers need to be biocompatible and bioresorbable in order to minimize adverse reactions in the organism [1]. The increasing therapeutic use of polymers as biomaterial implants makes it essential to evaluate the behavior of subcutaneous implants in vivo. This is generally done in rats, which provide an ideal model for analyzing the rate of neovascularization, the formation of collagen types
I and III, and the consolidation of the conjunctive capsule around the polymer [2].

Poly(L-lactide) is characterized by a high content of crystalline, high strength particles, and prolonged period of degradation. Poly(Dl lactide) is in contrast characterized by lower strength and quicker rates of degradation. Combining both poly(L-lactide) and poly(D,L lactide) results in a single amorphous copolymer that has a structure that combines the best characteristics of poly(L-lactic acid) and poly(DL-lactic acid), that is, the mechanical properties of the former and the shorter degradation time of the latter; these properties have made PLDLA a compound of great relevance in the controlled release of drugs and the treatment of bone fractures [3]. In addition to these applications, PLDLA serves as 
substrates for regenerative medicine applications with special focus on skin tissue engineering [4]. Despite these advantages, biomaterials containing poly(L-co-D,L lactic acid) are generally extremely dense, that is, devoid of pores and very rigid, and have a high modulus of elasticity, low elongation, and are hydrophobic; these characteristics limit the use of this polymer in a variety of medical applications, particularly when tissue/implant interactions are an important factor.

Many studies have shown that one way to improve the elongation characteristics, reduce the hydrophobicity of the polymer, and facilitate its interaction with tissue is to incorporate a low molecular weight polymer that can act as a plasticizer in polymeric matrices [5]. Polycaprolactone triol, a low molecular weight aliphatic polyester obtained by ring opening of $\varepsilon$-caprolactone to yield three $\mathrm{OH}$ groups in the monomer molecule of the polymer chain terminal, has been used as a plasticizer.

The aim of this work was to study the thermal, mechanical, morphological, and biological properties of PLDLA membranes containing different concentrations of poly(caprolactone triol) (PCL-T) as a plasticizer and to assess their potential usefulness in vivo.

\section{Experimental}

2.1. Materials. Poly(L-co-D,L lactic acid) (PLDLA; 70:30; $\mathrm{Mw}=282,700 \mathrm{~g} \cdot \mathrm{mol}^{-1}$ ) was synthesized via bulk ring-opening copolymerization of L-lactide and D, L lactide, using stannous octoate as the catalyst, essentially as described by Motta and Duek [6]. Poly (caprolactone triol) (PCL-T; average Mw $\left.=900 \mathrm{~g} \cdot \mathrm{mol}^{-1}\right)$, also known as 2-oxepanone, polymer with 2-ethyl-2-(hydroxymethyl)-1,3-propanodiol, was supplied by Solvay (CAPA 3091).

2.2. Membranes. PLDLA/PCL-T membranes were prepared by the solvent casting technique, in blends of 100/0, 90/10, 70/30, and 50/50\% (mass/mass), respectively, PLDLA/PCL-T in dichloromethane (Merck KgaA, Darmstadt, Germany), at a concentration of $10 \%\left(\mathrm{~m} \cdot \mathrm{vol}^{-1}\right)$. After complete dissolution of the copolymer, the solution was poured onto a glass plate and placed in a glass container that had been saturated with the solvent. After solvent evaporation, the membranes were dried under vacuum for $8 \mathrm{~h}$ and stored in a desiccator under vacuum until characterized.

\subsection{Characterization of PLDLA/PCL-T Membranes}

2.3.1. Dynamic Mechanical Analysis (DMA). DMA was done with a model 242 dynamic mechanical analyzer (Netzsch). The samples were subjected to deformation (amplitude: $240 \mu \mathrm{m})$ at a constant frequency of $1.00 \mathrm{~Hz}$ over a temperature range of $-100^{\circ} \mathrm{C}$ to $200^{\circ} \mathrm{C}$ and a heating rate of $5^{\circ} \mathrm{C} / \mathrm{min}$, using a traction system.

2.3.2. Differential Scanning Calorimetry (DSC). Calorimetric measurements were done with a DSC 2920 calorimeter (TAInstruments) in which 7-10 mg samples sealed in aluminum pans were heated from $25^{\circ} \mathrm{C}$ to $200^{\circ} \mathrm{C}$ at a rate of $10^{\circ} \mathrm{C} / \mathrm{min}$ and maintained at the final temperature for $5 \mathrm{~min}$ prior to cooling to $25^{\circ} \mathrm{C}$ at the same rate. The samples were maintained at $25^{\circ} \mathrm{C}$ for $5 \mathrm{~min}$ and then reheated. All tests were done under a nitrogen atmosphere.

2.3.3. Fourier Transform Infrared (FTIR) Spectroscopy. The quality of the PLDLA/PCL-T blends was assessed by FTIR spectroscopy. The IR measurements were done on PerkinElmer Spectrum One Perkin-Elmer 983G infrared spectrophotometer, using the $\mathrm{KBr}$ pellet technique, over the frequency range of $400-4000 \mathrm{~cm}^{-1}$, with a total of 256 scans.

2.3.4. Surface Contact Angle of the Membrane. The contact angles were measured with a contact angle goniometer (Ramé-Hart 100-00) and drop shape analysis software (RHI 2001 Imaging Software) at room temperature. A drop of deionized water $(0.2 \mathrm{~mL})$ was placed on the sample and the images of the water on the sample were recorded using a digital camera at 0.5 second intervals. From the angle measured between the droplet baseline and the tangent at the water/air boundary, a contact angle was calculated as the average of the measured left and right contact angles. For statistical analysis, three contact angle measurements were obtained.

Contact angle measurements were also used to evaluate the surface energy of the samples. This was done by measuring the angle $(\theta)$ to a polar liquid (water) and nonpolar liquid (diiodomethane) using the method harmonic [7].

2.3.5. Tensile Strength Test. PLDLA/PCL-T membranes containing polymer blends of 100/0, 90/10, 70/30, and 50/50 $(\mathrm{m} / \mathrm{m})$ were cut into strips $13.01 \pm 0.45 \mathrm{~mm}$ wide and approximately $0,20 \pm 0.45 \mathrm{~mm}$ thick and the tensile strength was determined with a model 810 Testar II apparatus (Instron) at $50 \mathrm{~mm} \cdot \mathrm{min}^{-1}$ and a load capacity of $1 \mathrm{kN}$. The samples were tested five times at a fixed humidity $(50 \%)$ and temperature $\left(25 \pm 2^{\circ} \mathrm{C}\right)$.

\subsection{Studies In Vivo with PLDLA/PCL-T Membranes (Blends 100/0 and 90/10)}

2.4.1. Animals. Six 3-month-old Wistar rats weighing $\sim 300 \mathrm{~g}$ were used. The rats were supplied by the Vivarium of the Pontifical Catholic University of São Paulo, the Center for Biological and Medical Sciences Sorocaba, and were housed at $22 \pm 2^{\circ} \mathrm{C}$ on a $12 \mathrm{~h} \mathrm{light/dark} \mathrm{cycle} \mathrm{with} \mathrm{food} \mathrm{and} \mathrm{water} a d$ libitum. The animal experiments were approved by an institutional Committee for Ethics in Animal Use CEUA/PUC-SP.

2.4.2. Membrane Implantation. The PLDLA/PCL-T membranes (blends 100/0 and 90/10) were immersed in 70\% ethanol and then vacuum-dried. The rats were randomly divided into two groups of three rats each, with one group receiving the 100/0 PLDLA/PCL-T membrane and the other receiving the 90/10 PLDLA/PCL-T membrane. The time intervals studied were $1,3,7,15,30$, and 60 days after implantation. 


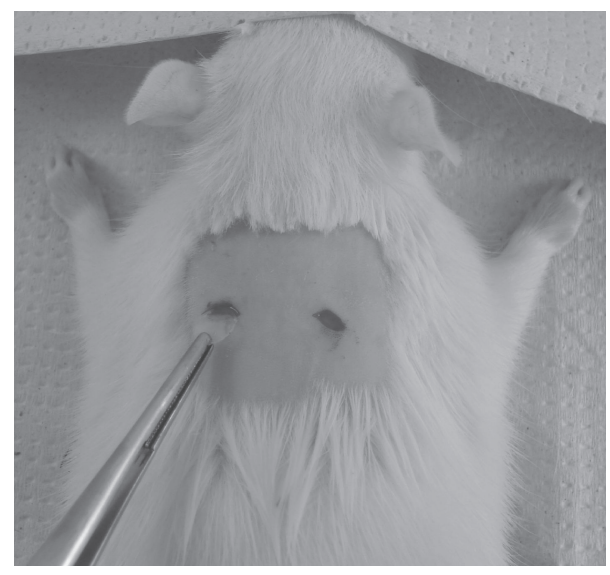

FIGURE 1: Surgical implantation of PLDLA/PCL-T membrane. A membrane disc was implanted in the incision on the left while the incision on the right served as the control.

The rats were anesthetized with a combination of ketamine and xylazine $(50 \mathrm{mg} / \mathrm{kg}$ and $10 \mathrm{mg} / \mathrm{kg}$, i.p., resp.). Subsequently, two lateral incisions approximately $1 \mathrm{~cm}$ long were made in the dorsal cervical region. A membrane disc $6 \mathrm{~mm}$ in diameter was inserted into the left incision while the right incision served as the control (sham operated, no membrane inserted) (Figure 1). One implant was placed in each rat. The incisions were then sutured with nylon 5-0 suture.

At each time interval after implantation, the rats were anesthetized with ketamine $10 \%(100 \mathrm{mg} / \mathrm{Kg})$ and xylazine $2 \%(6 \mathrm{mg} / \mathrm{Kg})$ and the tissues surrounding the implant were excised.

2.4.3. Histological Analysis. The material surrounding the tissues was washed in saline solution and immediately fixed in $10 \%$ paraformaldehyde. The specimens were dehydrated in an increasing alcohol series, embedded in a paraffin (Histosec, Merck) and sectioned ( $4 \mu \mathrm{m})$ with a high-precision microtome (Leica RM 2245). The sections were subsequently mounted and stained with hematoxylin and eosin (H\&E) and with Masson's trichrome (for 30- and 60-day intervals). The stained sections were examined with a Nikon Eclipse E800 light microscope and images were captured using a Nikon FDX 35 digital camera attached to the microscope.

\section{Results and Discussion}

3.1. Mechanical and Physicochemical Properties. DMA was done on membranes containing PLDLA/PCL-T blends of $100 / 0,90 / 10,70 / 30$, and 50/50. The $\alpha$-relaxation associated with the glass transition $\left(T_{g}\right)$ of the amorphous phases of PLDLA and PCL-T was determined from curves of loss of modulus versus temperature $\left(E^{\prime \prime}\right.$ versus $\left.T\right)$, as described in ASTMD 4065-2001. The $E^{\prime \prime}$ versus $T$ curves showed the presence of two glass transitions $\left(T_{g}\right)$ typical of the presence of PLDLA and PCL-T (Figure 2(a)). The $T_{g}$ values for PLDLA decreased significantly in membranes containing $10 \%$ and
TABLE 1: The $T_{g}$ temperatures for PLDLA/PCL-T membranes determined by DMA based on the loss of modulus $\left(E^{\prime \prime}\right)$.

\begin{tabular}{lcc}
\hline PLDLA/PCL-T blend & PLDLA $T_{g}\left({ }^{\circ} \mathrm{C}\right)$ & PCL-T $T_{g}\left({ }^{\circ} \mathrm{C}\right)$ \\
\hline $100 / 0$ & 38 & - \\
$90 / 10$ & 31 & -64 \\
$70 / 30$ & 28 & -70 \\
$50 / 50$ & 35 & -63 \\
\hline
\end{tabular}

30\% PCL-T (Table 1), indicating that the components were partially miscible. These findings agreed with those of Meier et al. (2004) [8] who used PCL-T as a plasticizer in cellulose acetate films and observed that the $T_{g}$ of cellulose acetate decreased as the PCL-T content increased.

The 50/50 blend (Figure 2(a)) showed behavior indicative of immiscibility (phase separation) of the components and this was confirmed by the $T_{g}$ values, which were very close to those of pure polymers; this immiscibility probably reflected the presence of excess plasticizer. Experiments with PLLA/PEG blends found that a plasticizer concentration > $20 \%$ caused phase separation [9].

Figure 2(a) shows that there was secondary $\beta$-type relaxation in PLDLA/PCL-T blend 100/0 $(\diamond)$ caused by the mobility of the methyl group linked to its main chain. This relaxation disappeared in the presence of PCL-T, probably because of the steric hindrance caused by PCL-T or overlap in the melting temperature of PCL-T.

Figure 2(b) shows a drop in the storage modulus $\left(E^{\prime}\right)$ for blends $90 / 10$ and 70/30, whereas blend 50/50 had a higher value than blends $90 / 10$ and $70 / 30$ at the start, but then decreased thereafter. The storage modulus of pure PLDLA declined at $37^{\circ} \mathrm{C}$ relative to the $T_{g}$ of this polymer. For blends 90/10, 70/30, and 50/50, the $T_{g}$ of the PLDLA phase was 31, 28 , and 37 , respectively, while the corresponding values for PCL-T were $-63,-66$, and $-59^{\circ} \mathrm{C}$ (Figure 2(b)).

3.2. Calculation of the Interaction Parameter $\left(\chi_{12}\right)$ for PLDLA/ $P C L-T$ Blends. The interaction parameters of the polymer blends were calculated based on the $T_{g}$ obtained by DMA. According to the Flory-Huggins theory, for a system to show complete miscibility, the interaction parameter $\left(\chi_{12}\right)$ should be $<0.5$; in this case only the $T_{g}[10]$. The $\chi_{12}$ for PLDLA/PCL$\mathrm{T}$ blends was calculated using the Fox equation as follows:

$$
\frac{1}{T_{g}}=\frac{\Phi_{1}^{\prime}}{T_{g 1}}+\frac{\Phi_{2}^{\prime}}{T_{g 2}}
$$

where $T_{g}=$ vitreous transition temperature of the blend (PLDLA/PCL-T), $\Phi_{1}^{\prime}=$ composition of component 1 (PLDLA) in the blend, $T_{g 1}=$ vitreous transition temperature of component 1 (PLDLA in the 100/0 blend), $\Phi_{2}^{\prime}=$ composition of component of 2 (PCL-T) in the blend, and $T_{g 2}=$ vitreous transition temperature of component 2 (PCL-T in the $0 / 100$ blend). 


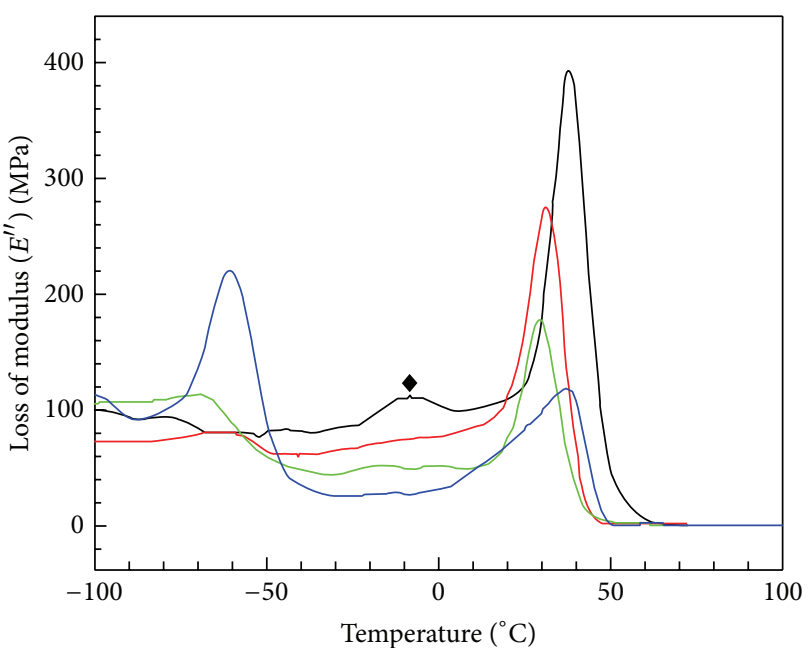

PLDLA/PCL-T

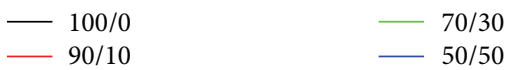

(a)
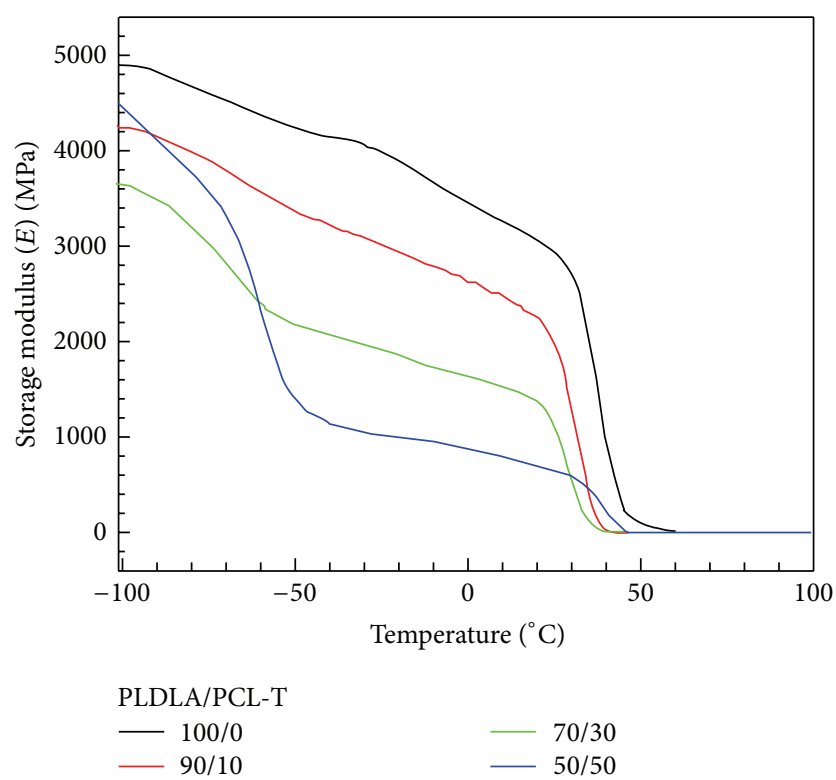

(b)

FIGURE 2: DMA curves for membranes containing PLDLA/PCL-T blends of 100/0, 90/10, 70/30, and 50/50. Panel (a): loss of modulus versus temperature ( $E^{\prime \prime}$ versus $T$ ); panel (b): storage modulus versus temperature ( $E^{\prime}$ versus $T$ ).

TABLE 2: Interaction parameters for different PLDLA/PCL-T blends.

\begin{tabular}{lccccc}
\hline PLDLA/PCL-T blend & $\Phi_{1}^{\prime}$ & $\Phi_{2}^{\prime}$ & $\Phi_{1}^{\prime \prime}$ & $\Phi_{2}^{\prime \prime}$ & $\chi_{12}$ \\
\hline $90 / 10$ & 0.96 & 0.04 & 0.09 & 0.91 & 0.6 \\
$70 / 30$ & 0.94 & 0.06 & 0.01 & 0.99 & 0.5 \\
$50 / 50$ & 0.98 & 0.02 & 0.09 & 0.90 & 0.8 \\
\hline
\end{tabular}

Based on the content of PLDLA and PCL-T, (2) [11] was used to obtain the interaction parameter of the blend $\left(\chi_{12}\right)$, the values of which are shown in Table 2:

$$
\begin{aligned}
\chi_{12}= & \left\{\left(\phi_{1}^{\prime 2}-\phi_{1}^{\prime \prime 2}\right)\left[\frac{m_{2} \ln \phi_{1}^{\prime \prime}}{\phi_{1}^{\prime}}+\left(m_{1}-m_{2}\right)\left(\phi_{2}^{\prime}-\phi_{2}^{\prime \prime}\right)\right]\right. \\
& \left.+\left(\phi_{2}^{\prime 2}-\phi_{2}^{\prime \prime 2}\right)\left[\frac{m_{1} \ln \phi_{2}^{\prime \prime}}{\phi_{2}^{\prime}}+\left(m_{2}-m_{1}\right)\left(\phi_{1}^{\prime}-\phi_{1}^{\prime \prime}\right)\right]\right\} \\
& \times\left(2 m_{1} m_{2}\left(\phi_{1}^{\prime 2}-\phi_{1}^{\prime \prime 2}\right) \cdot\left(\phi_{2}^{\prime 2}-\phi_{2}^{\prime \prime 2}\right)\right)^{-1},
\end{aligned}
$$

where $m_{1}=$ degree of polymerization of PLDLA $=1780.67$ and $m_{2}=$ degree of polymerization of PCL-T $=2.63$.

Table 2 shows that the addition of $10 \%, 30 \%$, and $50 \%$ PCL-T to the blends resulted in $\chi_{12}$ values of $0.6,0.5$, and 0.8 , respectively. The decrease in $\chi_{12}$ for the $70 / 30$ blend may be directly related to the higher concentration of polar components (plasticizer) that interact with the ester groups of PLDLA. The 50/50 blend had the highest $\chi_{12}$ compared to the other blends and was again indicative of the immiscibility of the system.

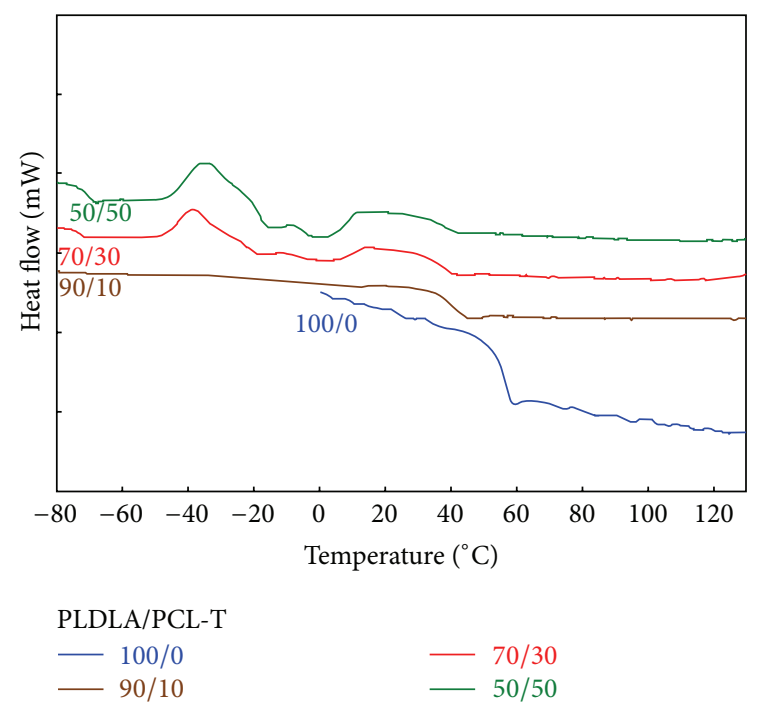

FIGURE 3: DSC curves for the second heating of membranes containing PLDLA/PCL-T blends of 100/0, 90/10, 70/30, and 50/50.

3.3. Differential Scanning Calorimetry (DSC). The DSC thermograms obtained in the second heating for PLDLA/PCL-T blends 100/0, 90/10, 70/30, and 50/50 are shown in Figure 3 and the results are summarized in Table 3.

There was no notable difference in the $T_{g}$ values for the first and second heating of membranes containing pure PLDLA (100/0 blend); there was also no melting transition phase in these curves, indicating that the material was amorphous, in case the membranes containing PCL-T has been a fall in the value of $T_{g}$ compared to pure PLDLA, which 
TABLE 3: DSC data for PLDLA/PCL-T membranes. The vitreous transition temperature $\left(T_{g}\right)$, crystallization temperature ( $\left.T_{c}\right)$, enthalpy of crystallization $\left(\Delta H_{c}\right)$, fusion temperature $\left(T_{f}\right)$, and enthalpy of fusion $\left(\Delta H_{f}\right)$ were obtained for the first and second heating.

\begin{tabular}{|c|c|c|c|c|c|c|c|}
\hline PLDLA/PCL-T blend & Heating & $\begin{array}{l}\text { PLDLA } \\
T_{g}\left({ }^{\circ} \mathrm{C}\right)\end{array}$ & $\begin{array}{l}\text { PCL-T } \\
T_{g}\left({ }^{\circ} \mathrm{C}\right)\end{array}$ & $\begin{array}{l}\text { PCL-T } \\
T_{c}\left({ }^{\circ} \mathrm{C}\right) \\
\end{array}$ & $\begin{array}{c}\text { PCL-T } \\
\Delta H_{c}(\mathrm{~J} / \mathrm{g})\end{array}$ & $\begin{array}{l}\text { PCL-T } \\
T_{f}\left({ }^{\circ} \mathrm{C}\right) \\
\end{array}$ & $\begin{array}{c}\text { PCL-T } \\
\Delta H_{f}(\mathrm{~J} / \mathrm{g})\end{array}$ \\
\hline $100 / 0$ & 1 st & 57 & $*$ & $*$ & $*$ & $*$ & $*$ \\
\hline $100 / 0$ & 2nd & 56 & $*$ & * & $*$ & $*$ & $*$ \\
\hline $90 / 10$ & 1 st & 51 & $*$ & * & * & $*$ & $*$ \\
\hline $90 / 10$ & 2nd & 41 & -74 & * & $*$ & & \\
\hline $70 / 30$ & 1 st & 48 & $*$ & $*$ & $*$ & $*$ & $*$ \\
\hline $70 / 30$ & 2nd & 38 & -73 & -38 & 6 & 3 & 2.5 \\
\hline $50 / 50$ & $1 s t$ & 55 & $*$ & $*$ & $*$ & $*$ & $*$ \\
\hline $50 / 50$ & $2 \mathrm{nd}$ & 38 & -71 & -33 & 16 & 2 & 4 \\
\hline Pure PCL-T & 2nd & $*$ & -71 & -21 & 20 & 5 & 4 \\
\hline
\end{tabular}

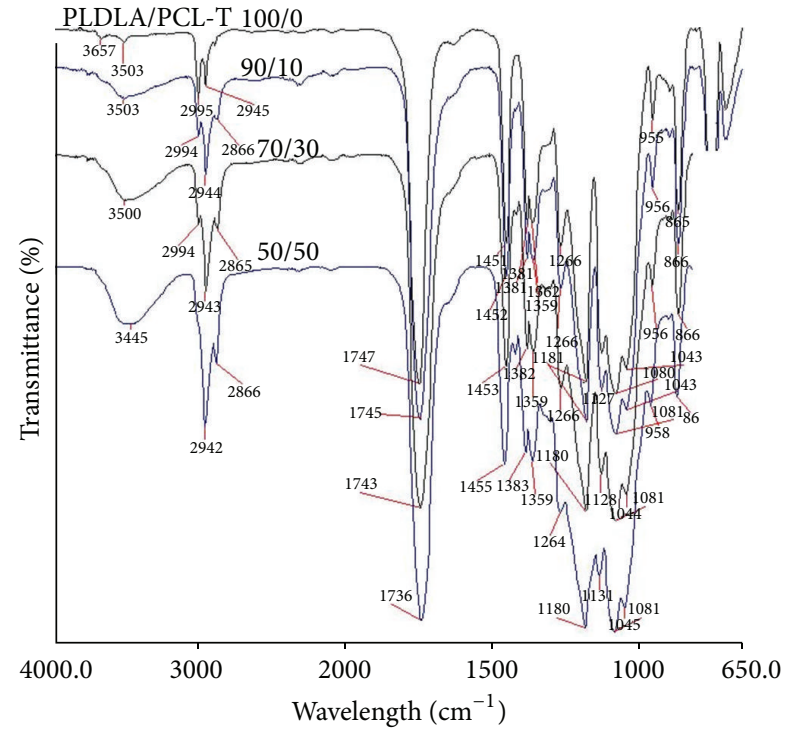

FIGURE 4: FTIR spectra for PLDLA/PCL-T membranes.

is a consequence of the increased flexibility of the material. The membranes of the blends $70 / 30$ and 50/50 showed similar behavior in their curves. The $T_{g}$ and crystallization temperature of PCL-T were well defined.

3.4. Fourier Transform Infrared (FTIR) Spectroscopy. The FITR spectra in Figure 4 show all the characteristic groups of the copolymer poly(L-D,L-lactide). The spectrum for the PLDLA membrane showed absorption bands $\left(n, \mathrm{~cm}^{-1}\right)$ at 2997-2965 $\left(\mathrm{CH}_{2}, \mathrm{CH}_{3}\right), 1747(\mathrm{C}=\mathrm{O}), 1360-1450\left(\mathrm{CH}_{3}\right), 1266$ (C-O bond in $\mathrm{COO})$, and $750(\mathrm{CH})$ characteristic of this material [12]. PCL-T showed bands similar to those of PLDLA that differed only in the axial asymmetrical deformation of fading in the $2995 \mathrm{~cm}^{-1} \mathrm{OH}$ stretch and the appearance of a peak in the terminal region of the polymer chains (around $3467 \mathrm{~cm}^{-1}$ ).

The FTIR spectra for PLDLA/PCL-T blends revealed a shift in the $\mathrm{OH}$ stretch in blends 90/10 and 70/30 at a wavelength of $3500 \mathrm{~cm}^{-1}$ with respect to pure PCL-T $\left(3467 \mathrm{~cm}^{-1}\right)$; this shift can be assigned to links (H-bridges) between PLDLA and PCL-T. The 50/50 blend showed a band shift in the $\mathrm{OH}$ stretch to a shorter wavelength and this change was probably associated with excess plasticizer that could result in competition between the polymer/linked plasticizer, plasticizer/plasticizer.

3.5. Contact Angles. Measurement of the contact angle between water and a film surface is one of the easiest ways to characterize the hydrophilicity/hydrophobicity of a film. Good hydrophilicity is beneficial for biocompatibility and flow of nutritional liquid in vivo [13]. Cells adhere to and spread easily on substrates that have moderate hydrophilicity compared to very hydrophilic or hydrophobic substrates [14]. For the present analysis, the values (limits) for defining hydrophilic/hydrophobic interactions were a contact angle of $65^{\circ}$ and a water adhesion tension $(\tau)$ of 30 dynes $\cdot \mathrm{cm}^{-1}$, as described by Lim et al. (2004) [15].

The water adhesion tension was calculated as

$$
\tau=\gamma \cdot \cos \theta
$$

where $\gamma=$ water surface tension $\left(72.8 \mathrm{dynes} \cdot \mathrm{cm}^{-1}\right)$ and $\theta=$ contact angle [15].

Surfaces with contact angles $<65^{\circ}$ and high values of $\tau$ were defined as hydrophilic, whereas surfaces high contact angles and low values of $\tau$, that is, low wettability, were considered to be hydrophobic. Table 4 shows the contact angles, surface energy, and water adhesion tension for PLDLA/PCL$\mathrm{T}$ membranes. The values of $\tau$ were calculated according to (3).

Based on the values shown in Table 4, the surface of PLDLA/PCL-T 100/0 membranes was hydrophobic and the addition of PCL-T made this more hydrophilic, as shown by the decrease in the contact angle. This effect was expected because of the presence of polar groups in PCL-T that increase the interaction with water, increasing the surface energy and water adhesion tension. Figure 5 shows the wettability of the membrane surface of different blends of PLDLA/PCL-T with deionized water; as can be seen, wettability increased proportionally to the amount of PCL-T, in agreement with the results in Table 4. 


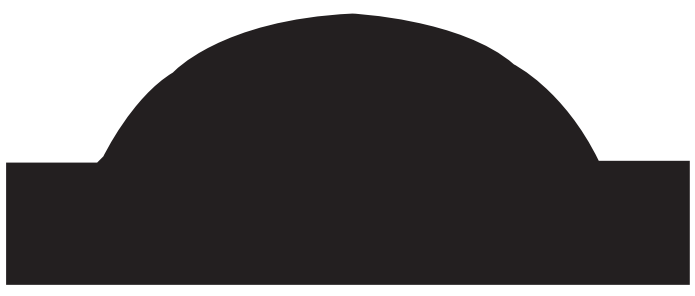

(a)

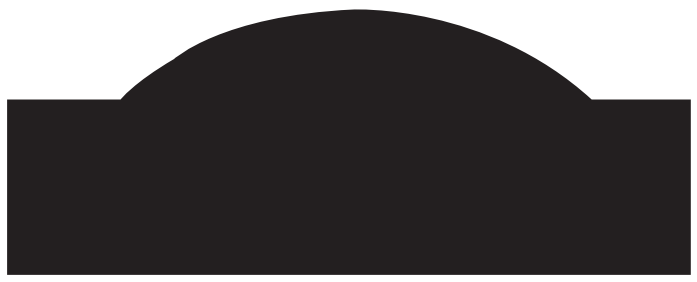

(c)

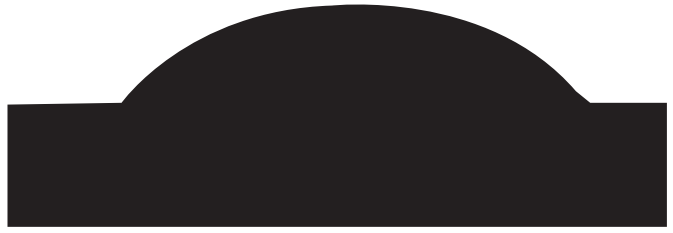

(b)

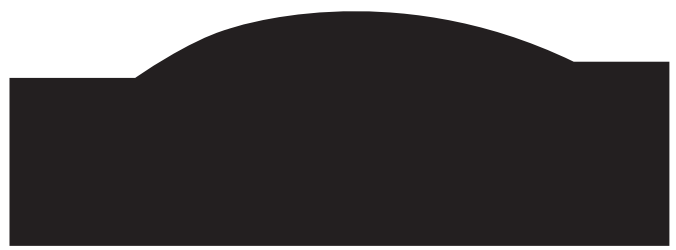

(d)

FiguRE 5: The change in the contact angles of membranes containing different blends of PLDLA/PCL-T: (a) 100/0, (b) 90/10, (c) 70/30, and (d) $50 / 50$.

TABLE 4: Contact angle, surface energy, and water adhesion tension for membranes containing PLDLA and PLDLA/PCL-T blends.

\begin{tabular}{|c|c|c|c|c|c|c|}
\hline \multirow[t]{2}{*}{ Blend } & \multicolumn{2}{|c|}{ Contact angle $(\theta)$} & \multicolumn{3}{|c|}{$\begin{array}{l}\text { Surface energy* } \\
\left(\mathrm{mJ} \cdot \mathrm{m}^{-2}\right)\end{array}$} & \multirow[t]{2}{*}{$\tau$ (dynes $\cdot \mathrm{cm}^{-1}$ ) } \\
\hline & $\mathrm{H}_{2} \mathrm{O}$ & $\mathrm{CH}_{2} \mathrm{I}_{2}$ & $\gamma_{s}^{p}$ & $\gamma_{s}^{d}$ & $\gamma_{s}$ & \\
\hline $100 / 0$ & 77 & 29 & 9 & 42 & 52 & 16 \\
\hline $90 / 10$ & 51 & 31 & 22 & 42 & 64 & 46 \\
\hline $70 / 30$ & 49 & 28 & 23 & 42 & 65 & 48 \\
\hline $50 / 50$ & 37 & 23 & 28 & 39 & 72 & 58 \\
\hline
\end{tabular}

3.6. Tensile Strength Tests. Table 5 and Figure 6 show the relationship between tensile properties and the PCL-T content of PLDLA/PCL-T membranes (100/0, 90/10, 70/30, and $50 / 50$ blends). PLDLA showed the typical characteristics of a vitreous polymer, with a modulus of elasticity of $1564 \pm$ $141 \mathrm{MPa}$, resistance to traction $\left(\sigma_{\max }\right)$ of $21 \pm 5.5 \mathrm{MPa}$, and deformation at rupture of $8.7 \pm 1.2 \%$ (Table 5). The addition of PCL-T significantly reduced the modulus of elasticity $(E)$ (Table 5), in agreement with the findings of Meier et al. (2004) $[8]$ in their study of the effects of PCL-T on cellulose acetate.

The presence of PCL-T significantly altered the deformation at rupture, indicating an increase in flexibility (Table 5, Figure 6); the 90/10 blend showed the greatest deformation at rupture, with a value of $21.4 \pm 6 \%$ compared to pure PLDLA, which had a value of $8.7 \pm 1.2 \%$. The higher value of the $50 / 50$ blend reflected the ability of the plasticizer to dissipate energy at fracture interfaces, thereby delaying rupture of the membrane [9]. With regard to the $70 / 30$ blend, one possible explanation for the unexpectedly higher modulus values obtained for this membrane compared to the $90 / 10$ membrane could be the proximity between the temperature

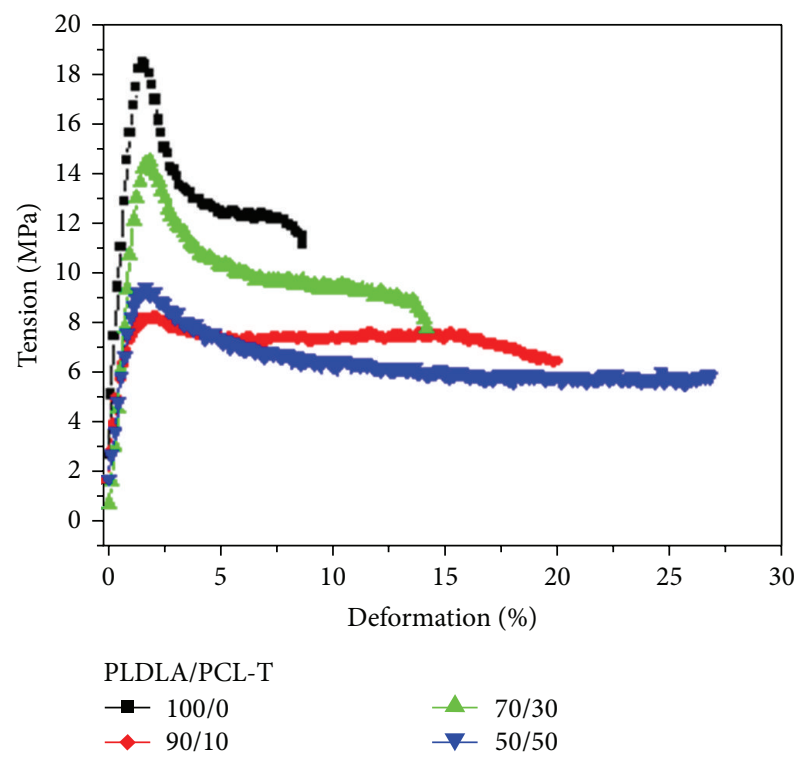

FIgURE 6: Tension versus deformation curves for PLDLA/PCL-T membranes. Standard deviation bars have been omitted for clarity.

at which the test was done $\left(25 \pm 2^{\circ} \mathrm{C}\right)$ and the $T_{g}\left(28^{\circ} \mathrm{C}\right)$ of PLDLA containing 30\% PCL-T.

\subsection{Histological Analysis of Implanted PLDLA/PCL-T Mem- branes (100/0 and 90/10 Blends)}

3.7.1. Hematoxylin-Eosin Staining. The inflammatory response on the first day after implantation was greater at the implanted sites than at control sites. Membranes containing PLDLA/PCL-T blend 100/0 showed fibrin deposition with polymorphonuclear neutrophils, as well as occasional eosinophils and mast cells (Figure 7(a)). A similar situation was observed for PLDLA/PCL-T blend 90/10 (Figure 7(b)). 
TABLE 5: Maximum tension, tension at rupture, deformation at rupture, and modulus of elasticity $(E)$ obtained in the tensile strength tests of PLDLA/PCL-T membranes.

\begin{tabular}{lcccc}
\hline $\begin{array}{l}\text { PLDLA/PCL-T } \\
\text { blend }\end{array}$ & $\begin{array}{c}\text { Maximum tension } \\
(\mathrm{MPa})\end{array}$ & $\begin{array}{c}\text { Tension at rupture } \\
(\mathrm{MPa})\end{array}$ & $\begin{array}{c}\text { Deformation at } \\
\text { rupture }(\%)\end{array}$ & $\begin{array}{c}\text { Modulus of } \\
\text { elasticity }(E)(\mathrm{MPa})\end{array}$ \\
\hline $100 / 0$ & $21 \pm 5.5$ & $9.7 \pm 1.8$ & $8.7 \pm 1.2$ & $1564 \pm 141$ \\
$90 / 10$ & $8.6 \pm 0.5$ & $6.5 \pm 0.7$ & $21.4 \pm 6.0$ & $795 \pm 198$ \\
$70 / 30$ & $15 \pm 0.8$ & $7.8 \pm 0.8$ & $13.5 \pm 2.0$ & $1016 \pm 198$ \\
$50 / 50$ & $9.2 \pm 0.2$ & $5.5 \pm 0.4$ & $26.8 \pm 2.0$ & $715 \pm 63$ \\
\hline
\end{tabular}

The values are the mean \pm SD of 5 determinations.

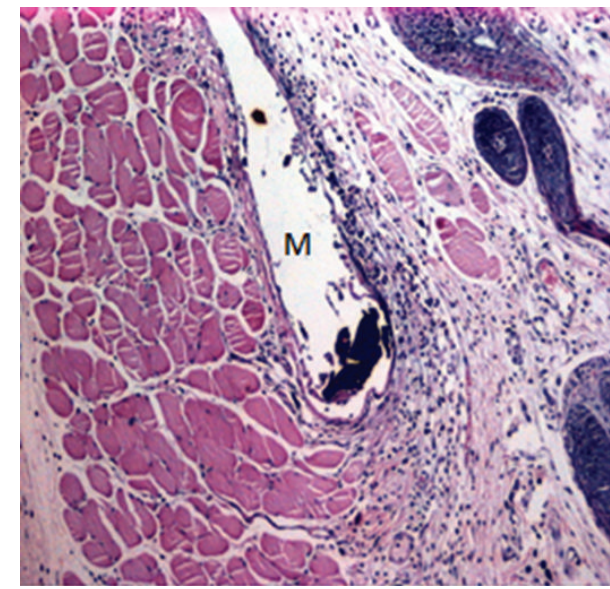

(a)

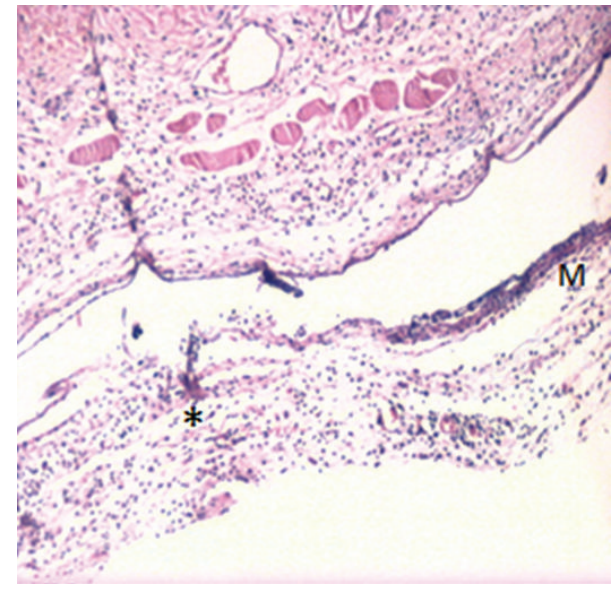

(b)

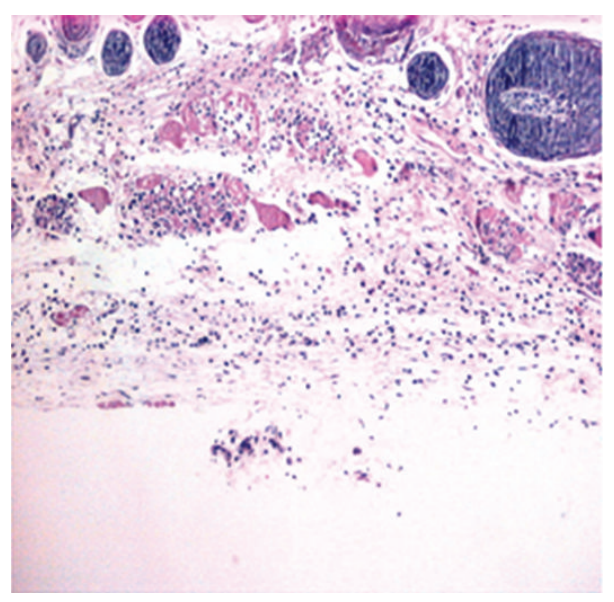

(c)

Figure 7: Photographs of sites one day after implantation of PLDLA/PCL-T membranes. (a) Site implanted with the 100/0 blend showing the formation of a fibrous capsule (*) around the membrane (M) and an infiltrate of polymorphonuclear cells, (b) site implanted with the 90/10 blend showing a similar result to (a), and (c) control site showing an infiltrate of polymorphonuclear cells. Staining: hematoxylin and eosin (H\&E). Magnification: 100x.

In all cases, the inflammatory infiltrate in the controls was markedly less than that at the implanted sites (Figure 7(c)).

At three days after implantation, sites implanted with the $100 / 0$ blend showed an area delimited by organized fibrin deposition, with congested vessels and the presence of mononuclear cells, but no giant multinucleated cells. Sites implanted with the 90/10 blend also showed the fibrin "capsule," but it was not as organized and there were frequently macrophages around the implant, in addition to congestion and edema. The control sites of both groups had a similar appearance, that is, granulated tissues with a chronic inflammatory infiltrate containing macrophages, plasmocytes, and mast cells.

At 15 days after implantation with the 100/0 blend, there was a well-defined capsule delimited by dense collagen and an internal surface containing a few mononuclear cells 


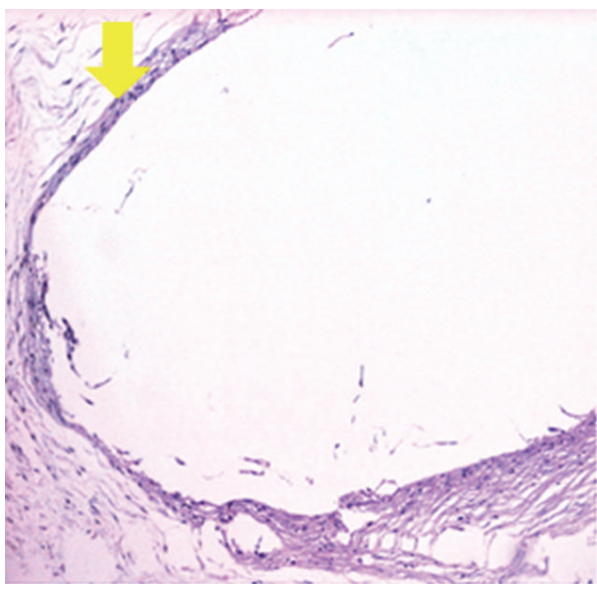

(a)

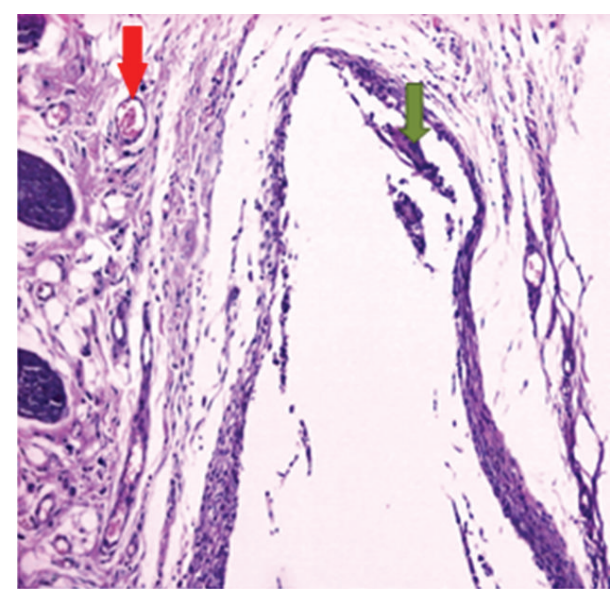

(b)

FIGURE 8: Photographs of sites 15 days after implantation of PLDLA/PCL-T membranes. (a) Site implanted with the 100/0 blend showing the formation of a capsule delimited by dense collagen (yellow arrow), (b) site implanted with the 90/10 blend showing individualized capsules and the presence of giant cells (green arrow) and blood vessels (red arrow). Staining: HE. Magnification: 100x.

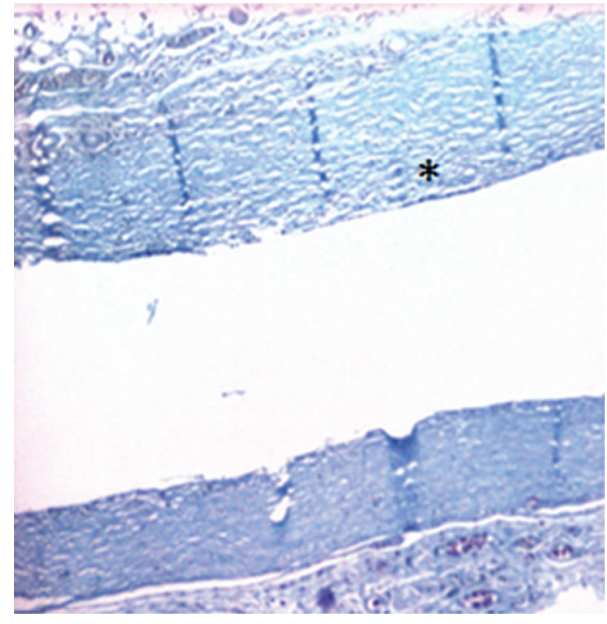

(a)

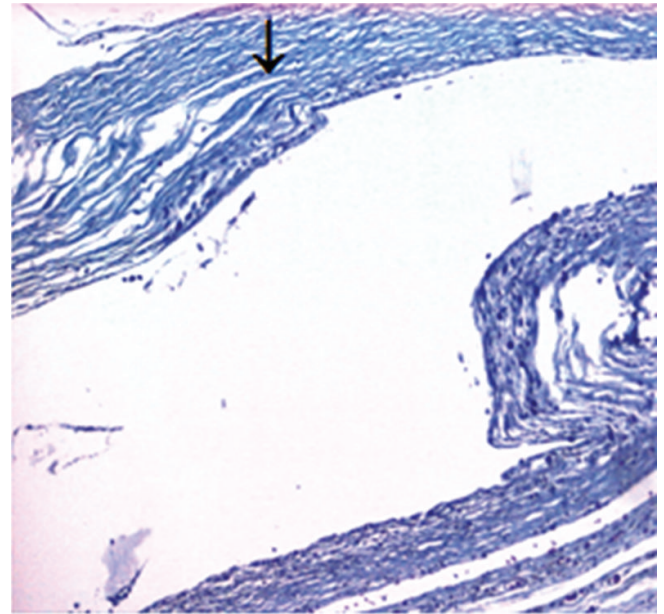

(b)

FIGURE 9: Photographs of sites 60 days after implantation of PLDLA/PCL-T membranes. (a) Site implanted with the 100/0 blend showing dense, well-organized collagen $(*)$, (b) site implanted with the $90 / 10$ blend showing mature, loosely organized collagen and little inflammatory infiltrate $(\downarrow)$. Staining: Masson’s trichrome. Magnification: 100x.

and hardly any giant cells (Figure 8(a)). At sites implanted with the 90/10 blend, the capsule occasionally contained giant foreign body cells, in addition to mononuclear cells (Figure 8(b)). All control sites showed complete healing with no significant alterations.

At 60 days after implantation, sites implanted with the 100/0 blend showed thick fibrosis around the capsule and practically no inflammatory infiltrate except for a small number of mononuclear cells in the interstitium (Figure 9). Sites implanted with the 90/10 blend showed a similar capsular organization to that seen with the 100/0 blend but with a discrete chronic inflammatory infiltrate; there were no giant multinucleated cells. The control sites showed no marked alterations. In contrast to our findings, previous studies of
PLLA membranes implanted subcutaneously in rats reporting the presence of giant multinucleated cells up to 180 days after the implantation reported that poly(DL-lactic acid) without plasticizer caused a chronic inflammatory response involving giant cells, macrophages with phagocytosed material, and fibroblast proliferation $[5,16]$. Macrophages have an important role in tissue repair through their ability to produce growth factors and mediators responsible for fibroblast proliferation and collagen synthesis [17].

3.7.2. Staining with Masson's Trichrome. The histological alterations observed with specific staining (Masson's trichrome) confirmed those observed with general staining (HE). In the case of the 100/0 blend, the capsule was practically devoid 
of an inflammatory infiltrate, while, in the case of the 90/10 blend, the collagen was looser and there was granulous tissue containing macrophages.

At 60 days after implantation, for both of the blends tested, there was well-formed, dense collagen that was practically devoid of an inflammatory infiltrate. No giant multinucleated cells were observed at any of the sites. For the 100/0 blend, the capsule was well organized with mature collagen and no inflammatory infiltrate (Figure 9(a)), while for the $90 / 10$ blend the collagen was more loosely organized and there were few mononuclear cells (Figure 9(b)).

Although this in vivo analysis was only a preliminary investigation, our results indicate that PLDLA membranes containing PCL-T are potentially useful, particularly since these polymers are nonallergic, nontoxic, and flexible; the latter characteristic in particular facilitates the handling of these membranes during surgical procedures.

\section{Conclusions}

In this study, we have shown that the addition of PCL-T to PLDLA membranes markedly affected the mechanical, thermal, morphological, physicochemical, and biological properties of the resulting membrane, especially increasing the flexibility of PLDLA. The addition of PCL-T at concentrations of $10 \%$ and $30 \%$ resulted in partially miscible systems while the addition of $50 \%$ resulted in immiscibility, probably because of the excess of plasticizer.

The analysis of PLDLA/PCL-T membranes in vivo indicated satisfactory polymer/tissue interaction. By 60 days after implantation, both 100/0 and 90/10 blends resulted in dense collagen deposition that was more loosely organized with the latter blend, probably because of the slower degradation.

These results indicate that the use of PLDLA PCL$\mathrm{T}$ membranes in medical devices could be advantageous, particularly where there is no need for long-term implants, for example, in supporting cell cultures, in dressing skin ulcers, and in guided regeneration in periodontics.

\section{Conflict of Interests}

The authors declare that there is no conflict of interests regarding the publication of this paper.

\section{References}

[1] M. Navarro, A. Michiardi, O. Castaño, and J. A. Planell, "Biomaterials in orthopaedics," Journal of the Royal Society Interface, vol. 5, no. 27, pp. 1137-1158, 2008.

[2] K. Lehle, S. Lohn, G. Reinerth, T. Schubert, J. G. Preuner, and D. E. Birnbaum, "Cytological evaluation of the tissue-implant reaction associated with subcutaneous implantation of polymers coated with titaniumcarboxonitride in vivo," Biomaterials, vol. 25, no. 24, pp. 5457-5466, 2004.

[3] D. Kim, A. Vaccaro, and R. G. Fessler, Spinal Instrumentation, chapter 37, Thieme Medical, New York, NY, USA; Wiley, New York, NY, USA, 2005.

[4] P. J. Kluger, R. Wyrwa, J. Weisser et al., "Electrospun poly(D/Llactide-co-L-lactide) hybrid matrix: a novel scaffold material for soft tissue engineering," Journal of Materials Science: Materials in Medicine, vol. 21, no. 9, pp. 2665-2671, 2010.

[5] L. C. V. Maluf-Meiken, D. R. M. Silva, E. A. R. Duek, and M. C. Alberto-Rincon, "Morphometrical analysis of multinucleated giant cells in subdermal implants of poly-lactic acid in rats," Journal of Materials Science: Materials in Medicine, vol. 17, no. 5, pp. 481-485, 2006.

[6] A. C. Motta and E. A. R. Duek, "Synthesis and characterization of the copolymer poly (L-co-D, L lactic acid)," Polímeros, vol. 17, no. 2, pp. 123-129, 2007.

[7] S. Wu, H. Butt, K. Graf, and M. Kappl, "Physics and chemistry of interfaces," Journal of Polymer Science Part C, vol. 34, p. 19, 1971.

[8] M. M. Meier, L. A. Kanis, J. C. de Lima, A. T. N. Pires, and V. Soldi, "Poly(caprolactone triol) as plasticizer agent for cellulose acetate films: influence of the preparation procedure and plasticizer content on the physico-chemical properties," Polymers for Advanced Technologies, vol. 15, no. 10, pp. 593-600, 2004.

[9] I. Pillin, N. Montrelay, and Y. Grohens, "Thermo-mechanical characterization of plasticized PLA: is the miscibility the only significant factor?" Polymer, vol. 47, no. 13, pp. 4676-4682, 2006.

[10] Polymer Handbook, Wiley-Interscience, New York, NY, USA, 4th edition, 2004.

[11] W. N. Kim and C. M. Burns, "Compatibility studies of blends of polycarbonate and poly(ethylene terephthalate)," Journal of Polymer Science, Part B: Polymer Physics, vol. 28, no. 9, pp. 14091429, 1990.

[12] K. Menemse and G. Demiz, "Synthesis, characterization and in vitro degradation of Poly(DL-Lactide)/Poly(DL-Lactide-coGlycolide)," Turkish Journal of Chemistry, vol. 13, pp. 153-161, 1993.

[13] Y. Wan, J. Yang, J. Yang, J. Bei, and S. Wang, "Cell adhesion on gaseous plasma modified poly-(L-lactide) surface under shear stress field," Biomaterials, vol. 24, no. 21, pp. 3757-3764, 2003.

[14] A. Tezcaner, K. Bugra, and V. Hasirci, "Retinal pigment epithelium cell culture on surface modified poly(hydroxybutyrate-cohydroxyvalerate) thin films," Biomaterials, vol. 24, no. 25, pp. 4573-4583, 2003.

[15] J. Y. Lim, X. Liu, E. A. Vogler, and H. J. Donahue, "Systematic variation in osteoblast adhesion and phenotype with substratum surface characteristics," Journal of Biomedical Materials Research A, vol. 68, no. 3, pp. 504-512, 2004.

[16] E. Solhein, B. Sudmann, G. Bang, and B. Sudmann, "Biocompatibility and effect on osteogenesis of poly(ortho ester) compared to poly(DL-lactic acid)," Journal of Biomedical Materials Research, vol. 49, no. 2, pp. 257-263, 2000.

[17] V. Kumar, A. K. Abbas, and N. Fausto, "Tissue renewal and repair: regeneration healing, and fibrosis," in Robbins and Cotran Pathologic Basis of Disease, V. Kumar, A. K. Abbas, and N. Fausto, Eds., p. 110, Elsevier Saunders, Philadelphia, Pa, USA, 7th edition, 2005. 

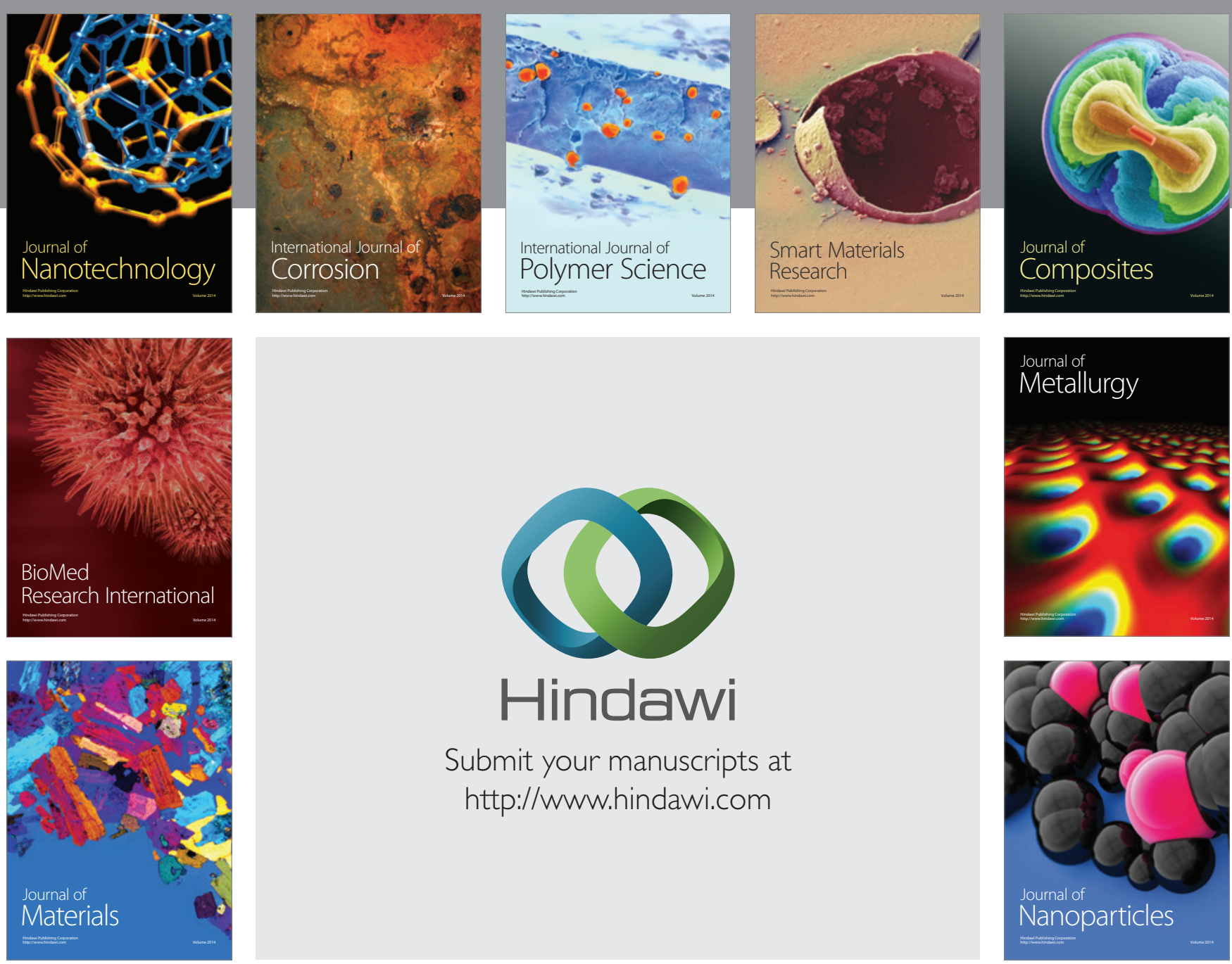

Submit your manuscripts at http://www.hindawi.com
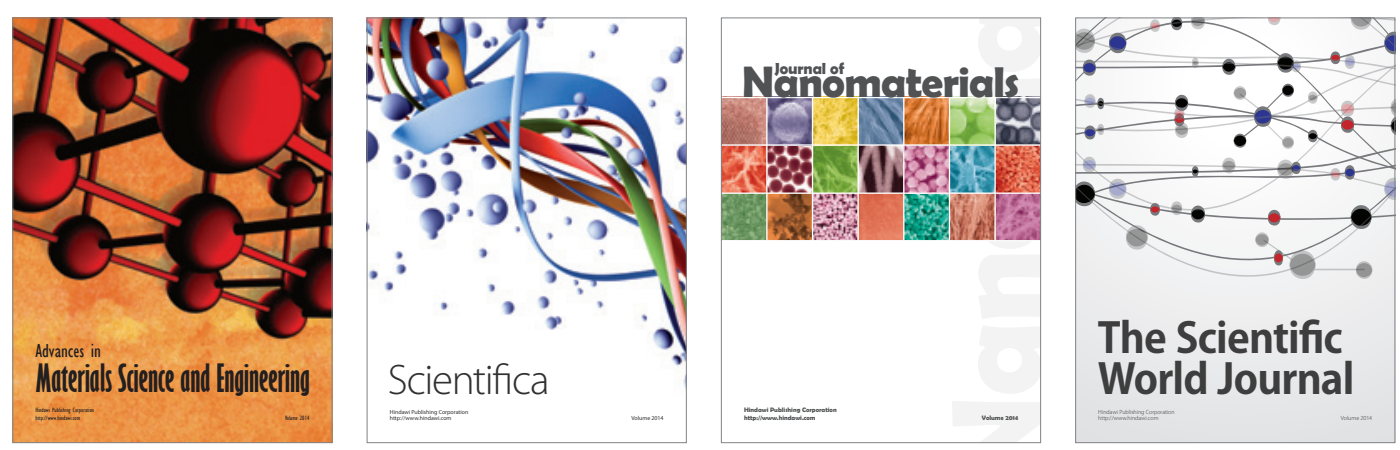

\section{The Scientific World Journal}
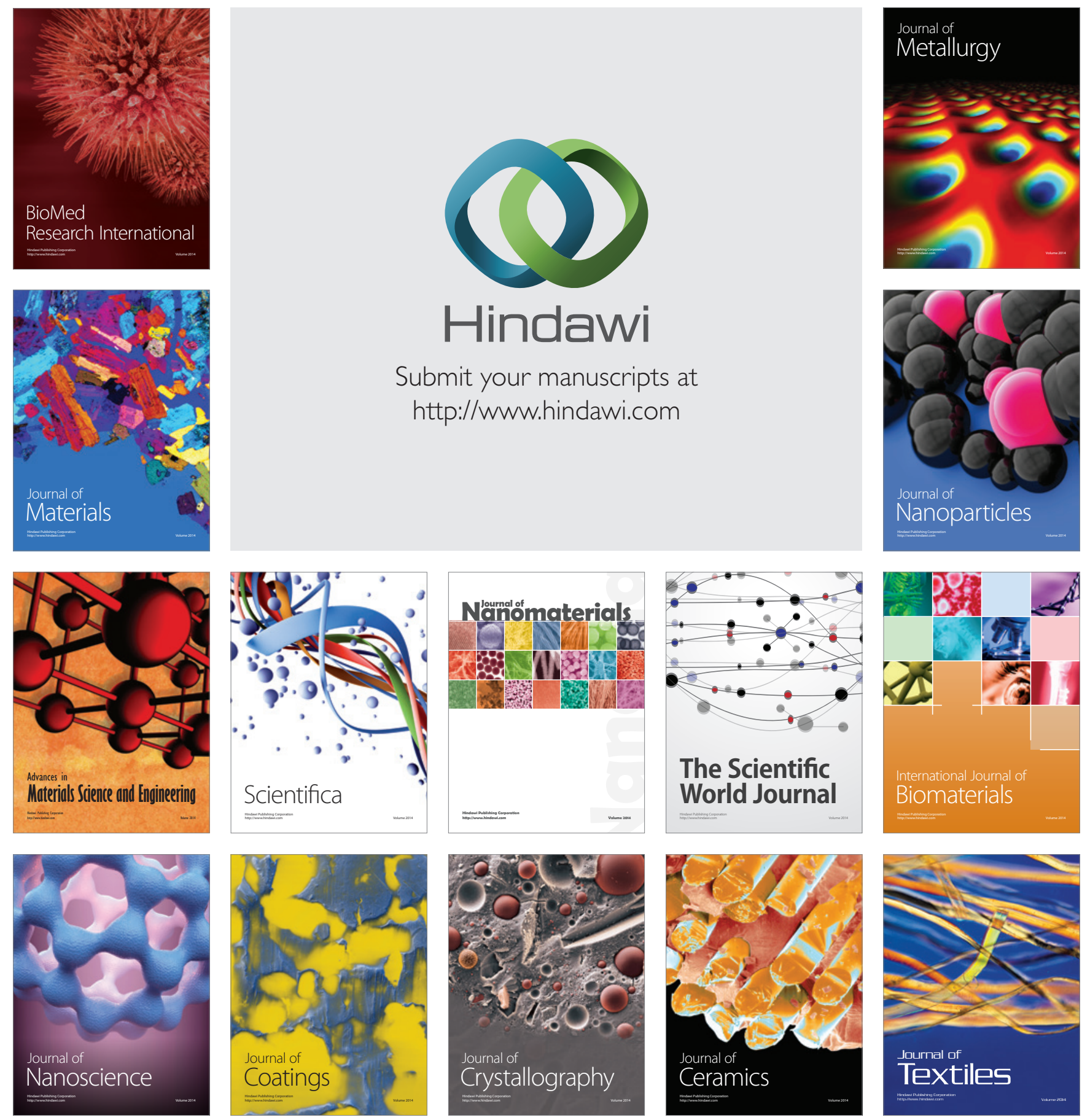